\title{
miR-96 exerts an oncogenic role in the progression of cervical cancer by targeting CAV-1
}

\author{
YONG CHEN $^{1}$, CHANGQING LIU ${ }^{1}$, BINGFAN XIE ${ }^{1}$, SHANGQIU CHEN $^{1}$, \\ YUAN ZHUANG ${ }^{1}$ and SHAOXIA ZHANG ${ }^{2}$ \\ Departments of ${ }^{1}$ Gynecology and ${ }^{2}$ Ultrasonography, The Fifth Affiliated Hospital of Sun Yat-sen University, \\ Zhuhai, Guangdong 519000, P.R. China
}

Received June 13, 2019; Accepted March 30, 2020

DOI: $10.3892 / \mathrm{mmr} .2020 .11101$

\begin{abstract}
Cervical cancer is the 4th most common malignant tumor type affecting women worldwide; however, its molecular mechanisms are not fully understood. Previous studies have indicated that microRNAs (miRs) serve crucial roles in the cellular functions of tumors. miR-96 is involved in the tumorigenesis of many cancer types. The aim of the present study was to investigate the role and mechanism of miR-96 in the progression of cervical cancer. The present results suggested that overexpression of miR-96 significantly enhanced the proliferative, migratory and invasive abilities of cervical cancer cells, while inhibiting miR-96 had the opposite effects. Additionally, activation of the Akt/mTOR signaling pathway was enhanced by miR-96 overexpression, while it was inhibited by the miR-96 inhibitor. Moreover, it was identified that miR-96 may directly target caveolin-1 (CAV-1) to decrease its expression level. Furthermore, overexpression of CAV-1 could reverse the increase in cell proliferation, migration and invasion induced by miR-96, as well as the upregulation of the Akt/mTOR signaling pathway. In conclusion, the present results suggested that miR-96 may have an oncogenic role in the progression of cervical cancer by targeting CAV-1. Therefore, miR-96 may be a potential target for cervical cancer therapy.
\end{abstract}

\section{Introduction}

Cervical cancer is the 4th most common malignant tumor in women worldwide, with $\sim 530,000$ incident cases and 270,000 mortalities each year (1). Infection with high risk human papillomaviruses (HPV) is a major risk factor for cervical cancer, while environmental factors such as smoking have also be

Correspondence to: Dr Changqing Liu, Department of Gynecology, The Fifth Affiliated Hospital of Sun Yat-sen University, 52 Meihua East Road, Zhuhai, Guangdong 519000, P.R. China E-mail: fuke8690@163.com

Abbreviations: CAV-1, caveolin 1; miRNA/miR, microRNA

Key words: cervical cancer, microRNA-96, migration, invasion shown to be correlated with cervical cancer occurrence (2). HPV-positive status can be detected in the majority of patients with cervical cancer and cervical malignant lesions, however $\sim 15 \%$ of patients are HPV-negative and cannot be detected by the HPV test kit (3). As the initial stage of cervical cancer is usually asymptomatic, many patients are diagnosed at an advanced stage, and thus the effect of surgical resection is limited (4). Therefore, it is important to identify novel targets in order to develop new therapeutic strategies for cervical cancer treatment.

Previous studies have indicated that microRNAs (miRNAs/miRs) serve crucial roles in the cellular features of tumors $(5,6)$. miRNAs are potential regulators of tumorigenesis and cancer development in various cancer types, including cervical cancer (5,7-9). miRNAs are expected to be used as a complementary treatment for cancer types due to the ability of miRNAs to target various physiological activities of cells, including proliferation, apoptosis and survival $(10,11)$. Moreover, several miRNAs have been identified to act as epigenetic drugs in glioblastoma, including miR-124, miR-101, miR-221 and miR-222 (12). Additionally, miRNAs not only act as cancer therapeutic targets, but also as promising biomarkers for diagnosis and prognosis (13). It has been previously demonstrated that miRNAs can be combined with chemotherapy agents for cancer therapy (14). The combination of miR-205 and gemcitabine can significantly inhibit the growth of gemcitabine-resistant pancreatic cancer cells (15), and the combination of miR-34a and paclitaxel improves anticancer activity in mice (16). An oncogenic role of miR-96 has been revealed in various cancer types such as breast cancer, hepatocellular carcinoma and prostate cancer (17-20). He et al (21) reported that miR-96 serves as a tumor suppressor in bladder cancer, while Ma et al (22) showed that miR-96 increases the proliferation and tumorigenicity of HeLa cells. However, the specific role of miR-96 in the metastasis of cervical cancer remains unknown.

Caveolin-1 (CAV-1) is an integral membrane protein that serves as major structural component of caveolae. CAV-1 is involved in cell adhesion and signal transduction, and has been shown to be involved in tumorigenesis (23-25). Zhou et al (26) reported that miR-124 regulates the progression of bladder cancer by targeting CAV-1. Furthermore, CAV-1 serves a role in clear cell renal cell carcinoma as a target of miR-124-3p (27). 
However, the role of CAV-1 in the progression of cervical cancer is not fully understood.

The aim of the present study was to investigate the role of miR-96 in the progression of cervical cancer. It was identified that overexpression of miR-96 promoted the proliferation, migration and invasion of the cervical cancer $\mathrm{SiHa}\left(\mathrm{HPV}^{+}\right)$ and C33A (HPV) cell lines, and also enhanced the Akt/mTOR signaling pathway. Moreover, the present results suggested that miR-96 may bind to CAV-1 mRNA, which indicates its possible oncogenic role in cervical cancer.

\section{Materials and methods}

Cell culture and transfection. The human cervical cancer $\mathrm{SiHa}\left(\mathrm{HPV}^{+}\right), \mathrm{C} 33 \mathrm{~A}\left(\mathrm{HPV}^{-}\right)$and $\mathrm{CaSki}\left(\mathrm{HPV}^{+}\right)$cell lines were obtained from the Cell Bank of the Chinese Academy of Sciences. Cells were cultured in RPMI-1640 medium (Thermo Fisher Scientific, Inc.) supplemented with $10 \%$ FBS (Gibco; Thermo Fisher Scientific, Inc.), penicillin (100 U/ml; Sigma-Aldrich; Merck KGaA) and streptomycin $\left(100 \mathrm{mg} / \mathrm{ml}\right.$; Sigma-Aldrich; Merck KGaA) at $37^{\circ} \mathrm{C}$ in an atmosphere of $5 \% \mathrm{CO}_{2}$. The pCMV-MIR-miR-96 (5'-UUUGGCACUAGCACAUUUUUGCU-3') vector was synthesized by Guangzhou RiboBio Co., Ltd., and was transfected into SiHa and C33A cells using Lipofectamine ${ }^{\circledR}$ 2000 (Invitrogen; Thermo Fisher Scientific, Inc.); the blank pCMV-MIR plasmid (Guangzhou RiboBio Co., Ltd.) was used as the negative control (NC). Another group of $\mathrm{SiHa}$ or C33A cells was co-transfected with pCMV-MIR-miR-96 and pcDNA-3.1-CAV-1 (Guangzhou RiboBio Co., Ltd.) or pcDNA-3.1-CAV-1 alone using Lipofectamine ${ }^{\circledR} 2000$ (Invitrogen; Thermo Fisher Scientific, Inc.). The miR-96 inhibitor (5'-AGCAAAAAUGUGCUAGUGCCAAA-3'; Guangzhou RiboBio Co., Ltd.) was transfected into CaSki cells using Lipofectamine ${ }^{\circledR} 2000$ (Invitrogen; Thermo Fisher Scientific, Inc.); miR control was used as the negative control. Transfection complexes were added to the medium at a final concentration of $50 \mathrm{nM}$. The cells were harvested for further experiments 24 or $48 \mathrm{~h}$ later.

Reverse transcription-quantitative PCR. After 24 h of transfection, cells were collected and extracted using a miRNA Purification kit (CoWin Biosciences) followed by RT with a miRNA cDNA Synthesis kit (cat. no. CW2141; CoWin Biosciences). RT was conducted at $42^{\circ} \mathrm{C}$ for $50 \mathrm{~min}$ and $85^{\circ} \mathrm{C}$ for $5 \mathrm{~min}$. qPCR was then performed using a SYBR-Green miRNA qPCR assay kit (cat. no. CW2142; CoWin Biosciences) according to the manufacturer's instructions. qPCR thermocycling conditions were as follows: Initial denaturation at $95^{\circ} \mathrm{C}$ for $10 \mathrm{~min}$, followed by 40 cycles of $95^{\circ} \mathrm{C}$ for $15 \mathrm{sec}$ and $60^{\circ} \mathrm{C}$ for $1 \mathrm{~min}$, and final extension at $72^{\circ} \mathrm{C}$ for $50 \mathrm{sec}$. Data were normalized using the endogenous U6. The primers used in this study were synthesized from Guangzhou RiboBio Co., Ltd. The U6 primer was 5'-CTC GCTTCGGCAGCACA-3' (sense). The miR-96 primer was 5'-TTTGGCACTAGCACATTTTTGCT-3' (sense). The anti-sense primers of miR-96 and U6 were obtained from the universal primers included in the SYBR-Green miRNA qPCR assay kit. The $2^{-\Delta \Delta C q}$ method was performed to analyze the RT-qPCR data (28).
Cell Counting Kit-8 (CCK-8) assay. Cells transfected with plasmids for $24 \mathrm{~h}$ were collected and seeded in a 96-well plate at a density of $1 \times 10^{3}$ cells per well at $37^{\circ} \mathrm{C}$ in a $5 \%$ $\mathrm{CO}_{2}$ atmosphere. At $0,24,48$ and $72 \mathrm{~h}, 10 \mu \mathrm{l}$ CCK-8 reagent (Beijing Solarbio Science \& Technology Co., Ltd.) was added to each well before the experiment according to the manufacturer's instructions, and incubated at $37^{\circ} \mathrm{C}$ for $1.5 \mathrm{~h}$. Then, the optical density value was measured using a microplate reader at a wavelength of $450 \mathrm{~nm}$, according to the manufacturer's protocol.

Colony formation assay. Cells were seeded into $60 \mathrm{~mm}$ dishes (500 cells/dish) following $24 \mathrm{~h}$ of transfection, and cultured for 1-2 weeks until visible colonies formed. The cells were fixed with $4 \%$ paraformaldehyde at room temperature for $30 \mathrm{~min}$ then stained with $0.1 \%$ crystal violet at room temperature for $30 \mathrm{~min}$, and the number of colonies was counted. Images were obtained using a camera.

Transwell assay. Transwell chambers (8- $\mu \mathrm{m}$ pore; EMD Millipore) in 24-well plates were performed for the assessment of cell migration and invasion abilities in vitro. Cells transfected with plasmids for $24 \mathrm{~h}$ were suspended in serum-free medium, then $1 \times 10^{5}$ cells were placed into the upper chamber pre-coated with or without Matrigel (BD Bioscience) for $1 \mathrm{~h}$ at $37^{\circ} \mathrm{C}$. Then, $500 \mu \mathrm{l}$ RPMI-1640 medium containing $10 \%$ FBS (Gibco; Thermo Fisher Scientific, Inc.) was added to the lower chamber and was cultured for $24 \mathrm{~h}$. The remaining cells in the upper chamber were removed with a cotton swab. Following washing with PBS, the cells were fixed with $4 \%$ polyformaldehyde for $30 \mathrm{~min}$ at room temperature and stained with $0.1 \%$ crystal violet for $20 \mathrm{~min}$ at room temperature. The number of migrated or invaded cells was counted and captured under a light microscope at magnification, x100 (Nikon Corporation).

Western blot analysis. After $48 \mathrm{~h}$ of transfection, RIPA lysis buffer (CoWin Biosciences) was used to extract cell proteins. Proteins were quantified using a bicinchoninic acid assay kit (Beijing Leagene Biotech Co., Ltd.). Protein samples (20 $\mu \mathrm{g})$ were resolved by $10 \%$ SDS-PAGE gel and transferred onto a PVDF membrane (EMD Millipore). Then, the membrane was blocked with $5 \%$ dried skimmed milk for $1 \mathrm{~h}$ at room temperature and probed with primary antibodies $(1: 1,000)$ at $4^{\circ} \mathrm{C}$ overnight. Antibody binding was detected with horseradish peroxidase-conjugated secondary antibodies $(1: 1,000$; ProteinTech Group, Inc.) for $1 \mathrm{~h}$ at room temperature and then chemiluminescence(Enhanced Chemiluminescence kit; CoWin Biosciences) was performed. GAPDH was used as the internal control, and the relative expression of proteins was normalized to GAPDH The densitometry was quantified using ImageJ software (v.1.48; National Institutes of Health). The primary antibodies used were as follows: Akt (cat. no. 10176-2-AP; ProteinTech Group, Inc.); phosphorylated(p)-Akt (Ser473; cat. no. 66444-1-Ig; ProteinTech Group, Inc.); mTOR (cat. no. 20657-1-AP; ProteinTech Group, Inc.); p-mTOR (Ser2448; cat. no. 2971; Cell Signaling Technology, Inc.); Cyclin D-1 (cat. no. 60186-1-lg; ProteinTech Group, Inc.); P70S6K (cat. no. 14485-1-AP; ProteinTech Group, Inc.); CAV-1 (cat. no. 16447-1-AP; ProteinTech Group, Inc.); and GAPDH (cat. no. 10494-1-AP; ProteinTech Group, Inc.). 
A

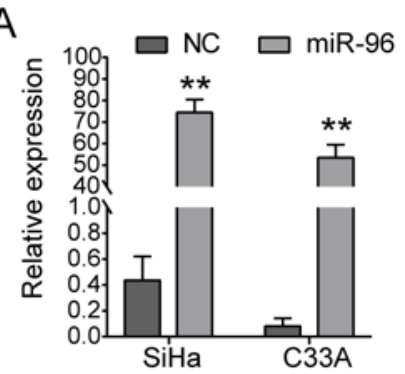

B

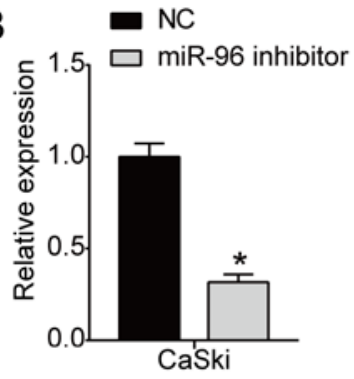

C

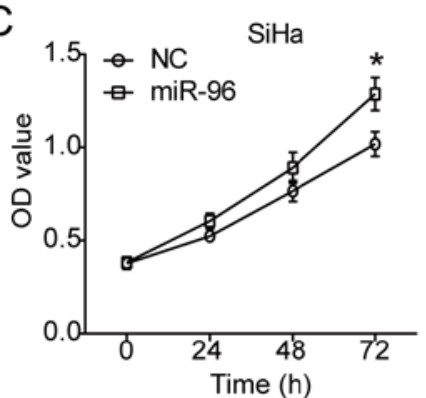

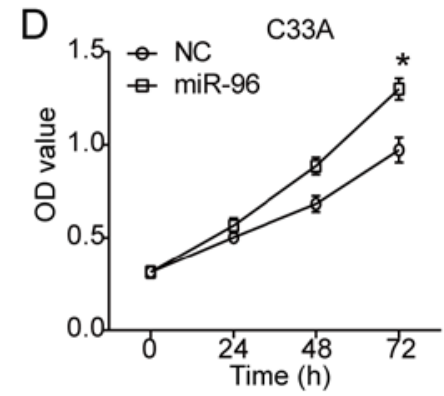

E
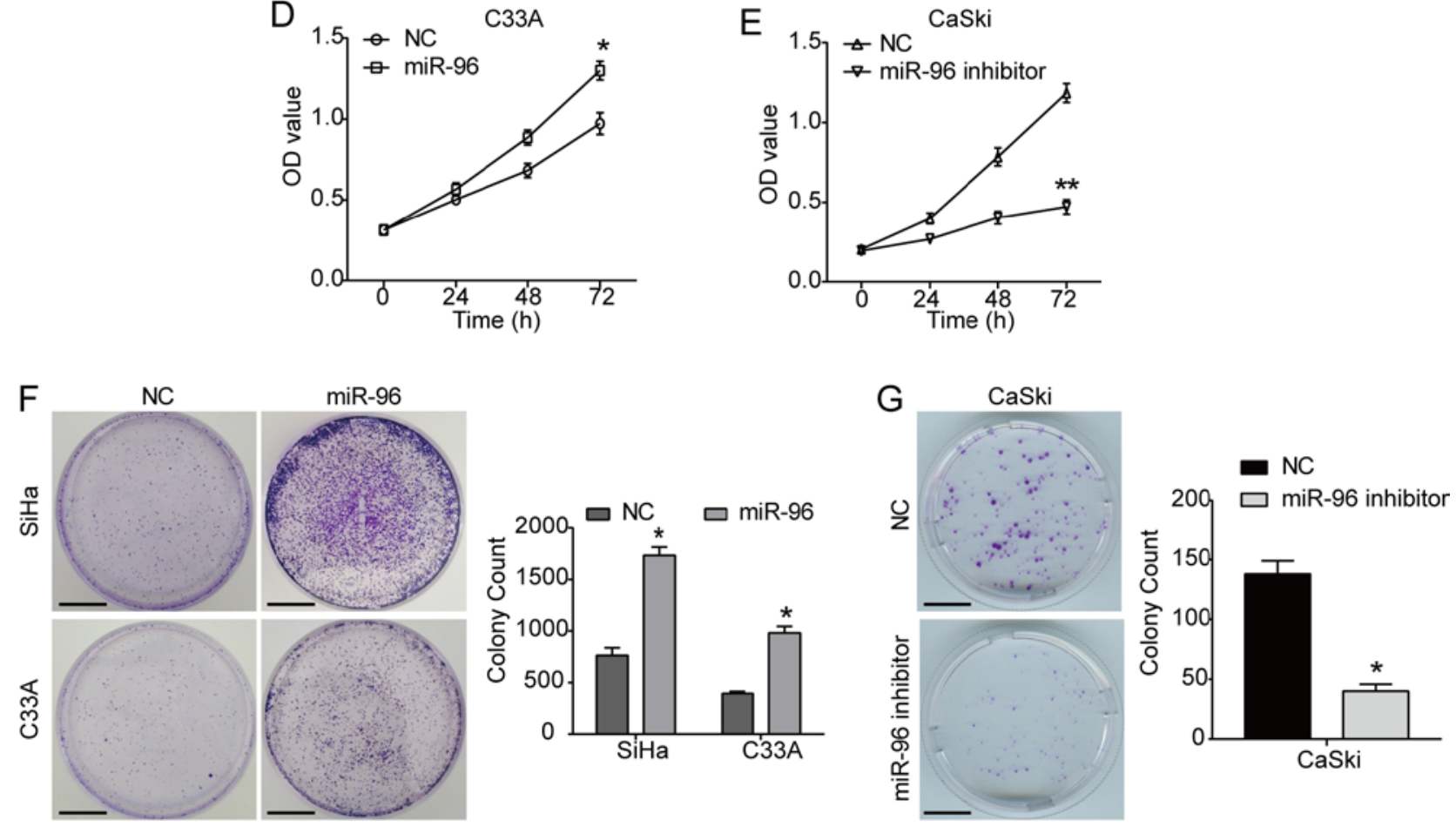

Figure 1. Overexpression of miR-96 promotes cell proliferation in cervical cancer. (A) Relative expression levels of miR-96 in SiHa and C33A cells transfected with pCMV-MIR-miR-96 vector. (B) Relative expression level of miR-96 in CaSki cells transfected with miR-96 inhibitor. A Cell Counting Kit-8 assay was performed to detect the viability of (C) SiHa, (D) C33A and (E) CaSki cells transfected with pCMV-MIR-miR-96 or miR-96 inhibitor. (F) A colony formation assay was used to examine the clonogenic capacities of SiHa and C33A cells transfected with the pCMV-MIR-miR-96 plasmid or NC. (G) A colony formation assay was performed to examine the clonogenic capacity of CaSki cells transfected with the NC or miR-96 inhibitor. Scale bar $=15 \mathrm{~mm}$. ${ }^{*} \mathrm{P}<0.05$ and ${ }^{* *} \mathrm{P}<0.01$. Data are presented as the mean \pm SD. miR-96, microRNA-96; NC, negative control; OD, optical density.

Dual-luciferase reporter assay. The potential target gene of miR-96 was predicted using the online software StarBase (v2.0; http://starbase.sysu.edu.cn/starbase2/index.php) (29). The wild-type (wt) or mutated (mut) CAV-1 3 ' untranslated regions (UTRs) were cloned into the pmirGLO plasmids (Promega Corporation). Cells were co-transfected with the pmirGLO-CAV-1-wt $(2 \mu \mathrm{g})$ or pmirGLO-CAV-1-mut $(2 \mu \mathrm{g})$ plasmid and pCMV-MIR-miR-96 using Lipofectamine ${ }^{\circledR} 2000$ (Invitrogen; Thermo Fisher Scientific, Inc.), and the blank plasmid was used as the negative control. After transfection for $48 \mathrm{~h}$, the luciferase activity was examined using a Dual-Luciferase Reporter assay system (Promega Corporation) according to the manufacturer's protocol. The firefly luciferase expression was normalized to Renilla luciferase activity.

Statistical analysis. Data are presented as the mean \pm SD from three independent experiments. GraphPad Prism 7.0 software (GraphPad Software, Inc.) was used for statistical analyses.
Group comparisons were analyzed with a Student's t-test or one-way ANOVA analysis followed by the Newman-Keuls method. $\mathrm{P}<0.05$ was considered to indicate a statistically significant difference.

\section{Results}

Overexpression of miR-96 enhances the proliferation of cervical cancer cells. To investigate the functional role of miR-96 in the progression of cervical cancer, the pCMVMIR-miR-96 vector was transfected into SiHa and C33A cells, which had an initial low miR-96 expression level, to overexpress miR-96; the blank pCMV-MIR vector was used as the NC (Figs. 1A and S1A). Additionally, due to the innate high miR-96 expression level in CaSki cells, CaSki cells were transfected with miR-96 inhibitor to downregulate miR-96 (Figs. 1B and S1A). It was identified that the overexpression of miR-96 significantly increased the viability of SiHa cells 

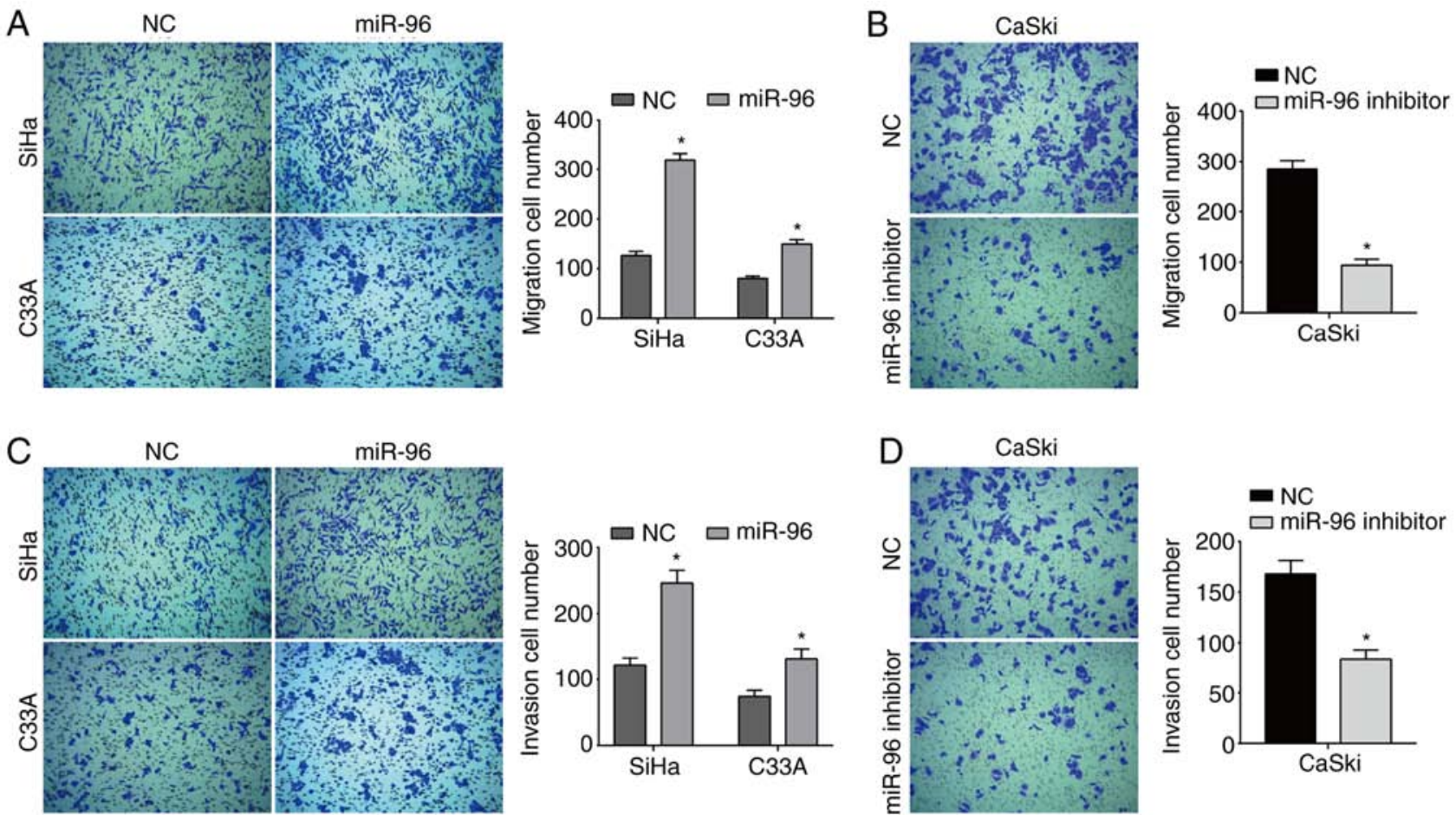

Figure 2. Overexpression of miR-96 enhances cell migration and invasion in cervical cancer. (A) Cell migration was detected using a Transwell assay after $\mathrm{SiHa}$ and C33A cells were transfected for $24 \mathrm{~h}$ with NC or pCMV-MIR-miR-96. (B) Migration of CaSki cells transfected with miR-96 inhibitor. (C) Invasion of SiHa and C33A cells was assessed using a Transwell assay. (D) Invasion of CaSki cells transfected with miR-96 inhibitor or NC. Original magnification, $\mathrm{x} 100 .{ }^{*} \mathrm{P}<0.05$. Data are presented as the mean \pm SD. miR-96, microRNA-96; NC, negative control.

compared with the NC group (Fig. 1C). Moreover, the viability of C33A cells was also promoted by miR-96 overexpression (Fig. 1D), whereas the miR-96 inhibitor significantly inhibited the viability of CaSki cells (Fig. 1E). In addition, the colony formation assay results indicated that miR-96 overexpression significantly increased the clonogenic capacities of both $\mathrm{SiHa}$ and $\mathrm{C} 33 \mathrm{~A}$ cells, while the miR-96 inhibitor had the opposite effect on CaSki cells (Fig. 1F and G).

miR-96 overexpression increases the migratory and invasive abilities of cervical cancer cells. The present results suggested that overexpression of miR-96 significantly increased cell migration in both $\mathrm{SiHa}$ and C33A cells (Fig. 2A). Furthermore, a significant decrease in cell migration was demonstrated in CaSki cells transfected with the miR-96 inhibitor (Fig. 2B). Moreover, miR-96 overexpression also increased the number of invaded cells compared with the NC group (Fig. 2C), while the miR-96 inhibitor reduced the invasive ability of CaSki cells (Fig. 2D).

miR-96 increases activation of the Akt/mTOR signaling pathway in cervical cancer cells. The Akt/mTOR signaling pathway serves an essential role in cellular processes, including cell proliferation, migration, survival and apoptosis. It was identified that there was a significant increase in the levels of p-Akt and p-mTOR in SiHa and C33A cells transfected with the pCMV-MIR-miR-96 vector, while the total expression of Akt and mTOR was not affected (Fig. 3A-C). In addition, the expression levels of the downstream proteins cyclin D1 and P70S6k were significantly increased by miR-96 overexpression in both SiHa and C33A cells (Fig. 3A-C). Moreover, it was demonstrated that the miR-96 inhibitor significantly inhibited the Akt signaling pathway by suppressing the expression levels of p-Akt and p-mTOR in CaSki cells (Fig. 3A and D). Therefore, the present results suggested that the Akt/mTOR signaling pathway may be involved in the oncogenic role of miR-96 in cervical cancer.

miR-96 directly targets $C A V-1$ in cervical cancer cells. Using the bioinformatics database, the present study identified that miR-96 has a conserved binding site in the 3'UTR of CAV-1 mRNA (Fig. 4A). In order to investigate this further, the pCMV-MIR-miR-96 vector or control vector was co-infected with CAV-1 3'UTR-wt or CAV-1 3'UTR-mut into $\mathrm{SiHa}$ and C33A cells. It was identified that miR-96 overexpression could significantly decrease the luciferase activity of the CAV-1 3'UTR-wt, while the luciferase activity of the CAV-1 3'UTR-mut was unaffected by miR-96 overexpression (Fig. 4B and C), suggesting that CAV-1 may be a target of miR-96. Moreover, overexpression of miR-96 significantly decreased the protein expression level of CAV-1 compared with the NC group (Fig. 4D). Collectively, the present results indicated that miR-96 may directly target CAV-1 and inhibit its expression in cervical cancer.

miR-96 increases cell proliferation, migration and invasion by targeting $C A V-1$. To further investigate the molecular mechanism of miR-96, SiHa and C33A cells were either transfected with pcDNA-3.1-CAV-1 vector alone (Fig. S1B and C) or co-transfected with pCMV-MIR-miR-96. The CCK-8 results suggested that the overexpression of CAV-1 significantly inhibited the viabilities of $\mathrm{SiHa}$ and C33A cells compared 
A

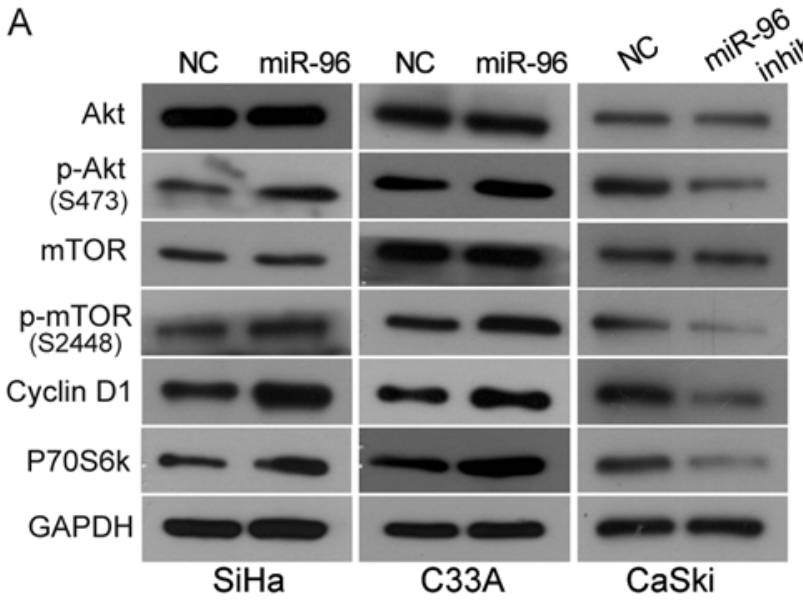

C

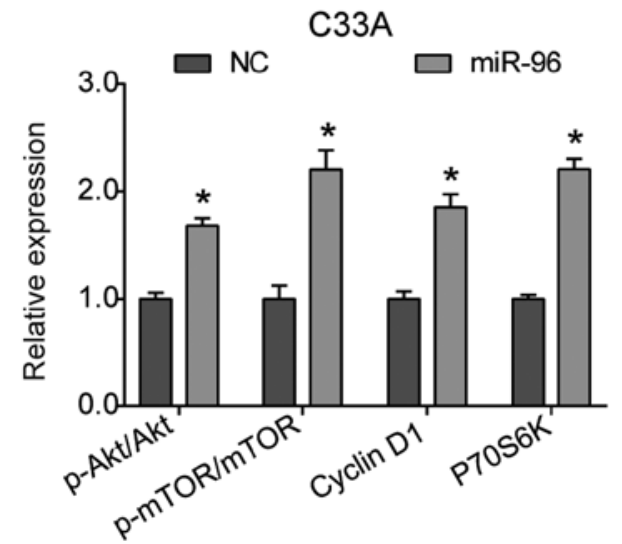

B

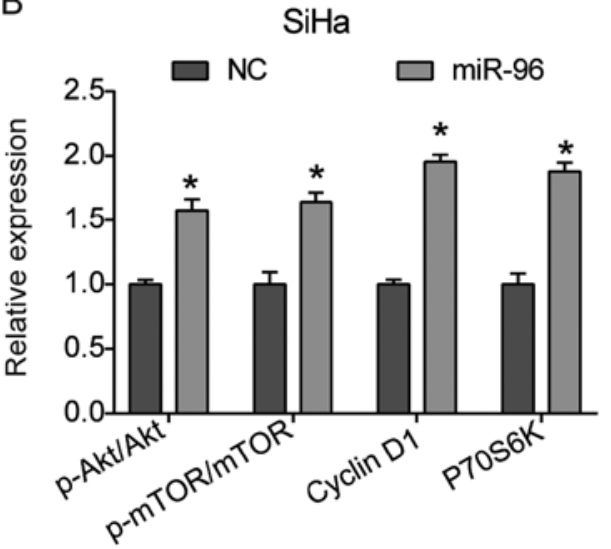

D

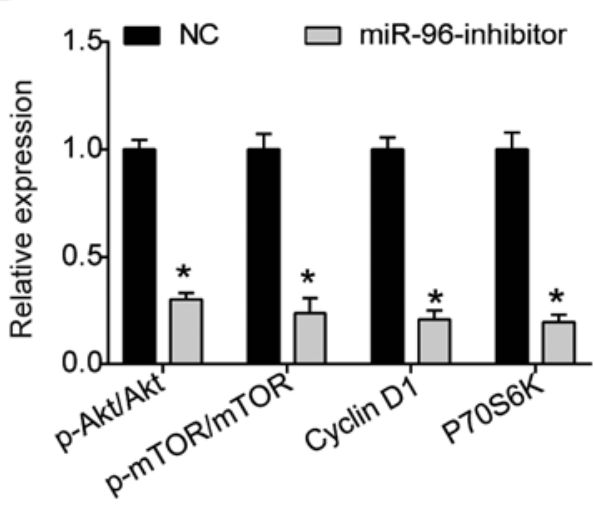

Figure 3. Overexpression of miR-96 promotes the activation of the Akt/mTOR signaling pathway in cervical cancer. (A) After $48 \mathrm{~h}$ transfection, western blot analysis was performed to detect the protein expression level of Akt/mTOR signaling pathway-associated proteins. Quantitative analysis of western blot analysis results in (B) SiHa, (C) C33A and (D) CaSki cells. " $\mathrm{P}<0.05$. Data are presented as the mean \pm SD. miR-96, microRNA-96; NC, negative control; p-, phosphorylated.

A miR-96 3' ucguuUUUACACGAUCACGGUUu 5'

CAV1 3'UTR-wt 39 5' cuuuUAAUUUUCCUGGUGCCAAu 3' 62 CAV1 3'UTR-mut $\quad$ 5'cuuuAAUUUUCCUGCACGGUUu 3'

C

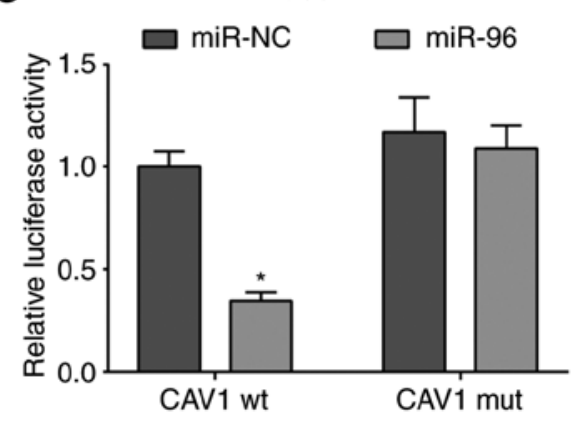

B

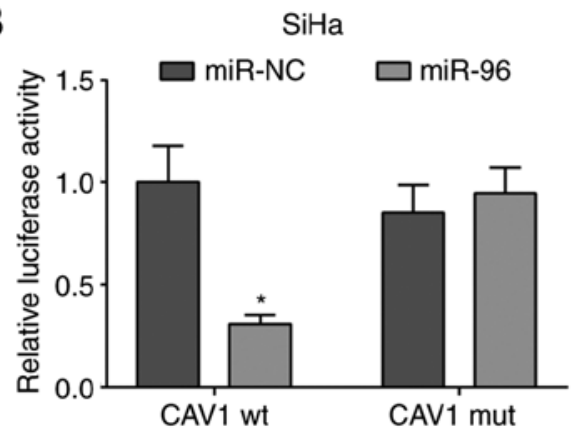

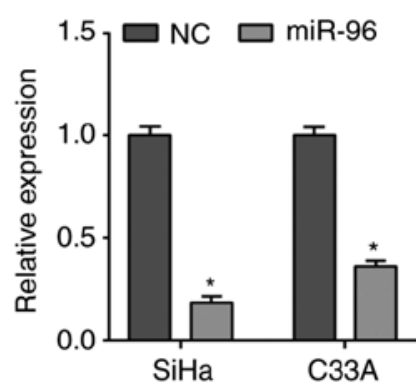

Figure 4. miR-96 directly targets CAV-1 and negatively inhibits its expression in cervical cancer. (A) Conserved binding site in the CAV-1 mRNA 3'UTR . Dual-luciferase reporter assays in (B) SiHa and (C) C33A cells co-infected with CAV-1-wt 3'-UTR or the CAV-1- mut 3'-UTR, with pCMV-MIR-miR-96 or pCMV-MIR. (D) Protein expression level of CAV-1 in cells transfected with pCMV-MIR-miR-96 or pCMV-MIR. "P<0.05. Data are presented as the mean \pm SD. miR-96, microRNA-96; NC, negative control; CAV-1, caveolin 1; 3'UTR, 3'untranslated region; wt, wild-type; mut, mutant. 
A

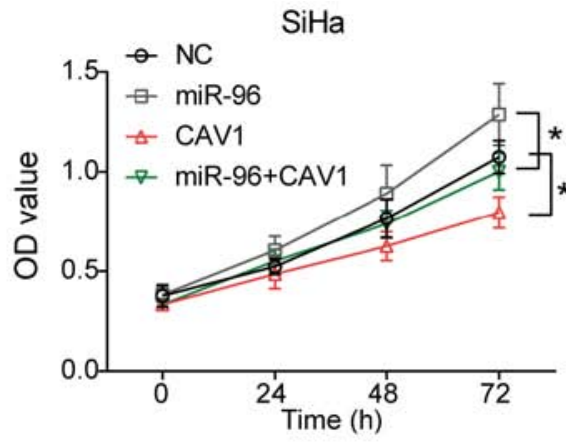

B

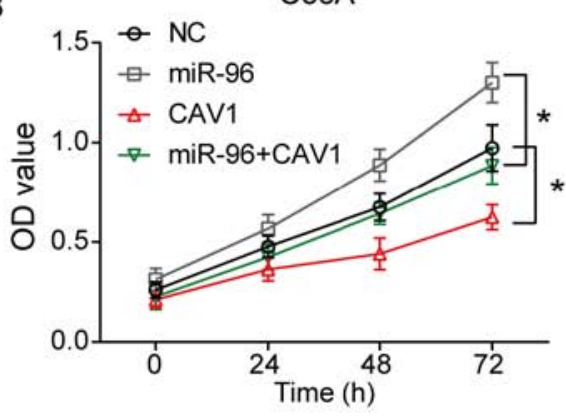

C NC

miR-96

CAV1
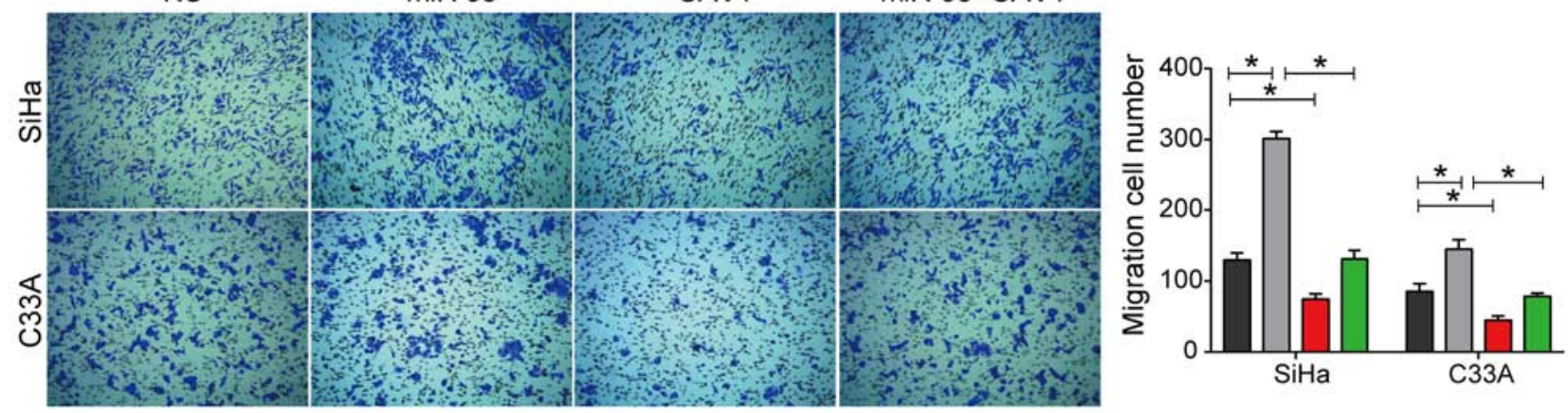

D NC

NC miR-96

CAV1

miR-96+CAV1
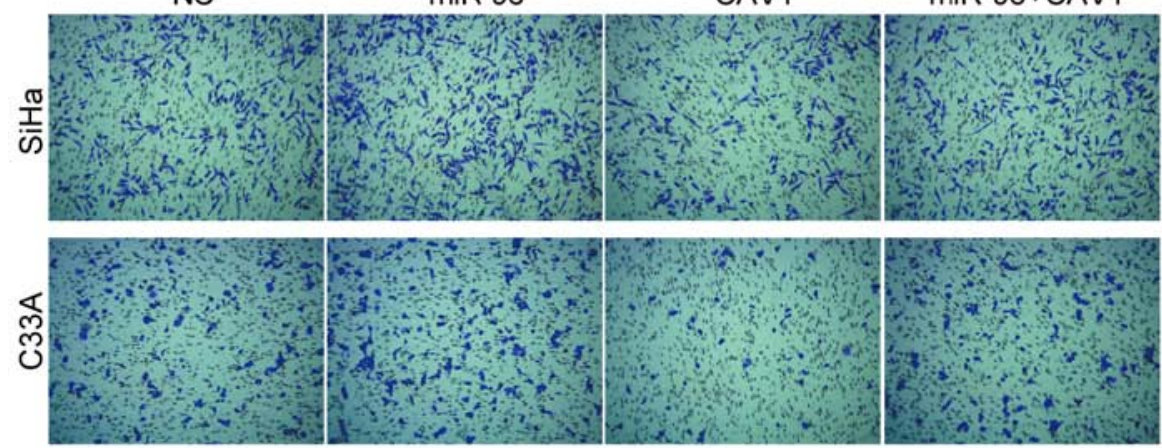

- NC $\square$ miR-96

$\square$ CAV1 $\square$ miR-96+CAV1

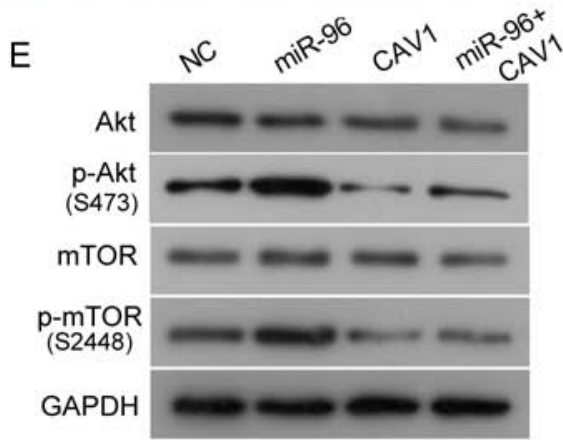

$\mathrm{F}$

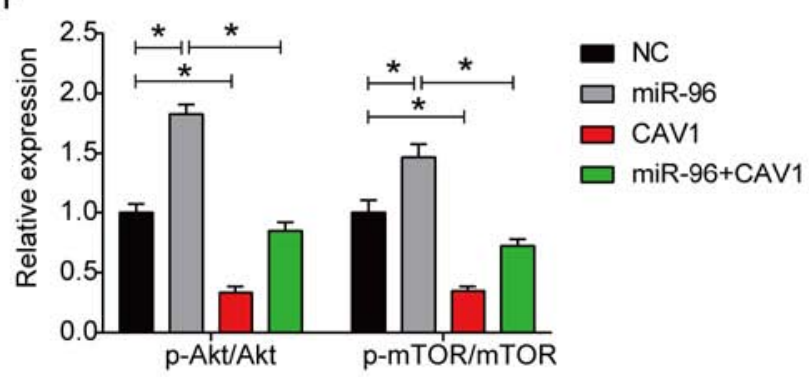

Figure 5. miR-96 promotes cell proliferation, migration and invasion in cervical cancer by targeting CAV-1. (A) SiHa and (B) C33A cells were co-transfected with miR-96 and CAV-1, or transfected with miR-96/CAV-1 alone, and then a Cell Counting-Kit 8 assay was used to detect cell viability. Following transfection for $24 \mathrm{~h}$, a Transwell assay was performed to assess (C) cell migration and (D) invasion. Original magnification, x100. (E) Protein expression levels of the Akt signaling pathway-associated proteins in SiHa cells. (F) Quantitative analysis of western blot analysis. *P<0.05. Data are presented as the mean \pm SD. miR-96, microRNA-96; NC, negative control; CAV-1, caveolin 1; OD, optical density; p-, phosphorylated.

with control cells, suggesting an inhibitory effect of CAV-1 on cell proliferation (Fig. 5A and B). Moreover, co-transfection of CAV-1 and miR-96 decreased cell viability compared with miR-96 alone (Fig. 5A and B). The Transwell assay results suggested that the overexpression of CAV-1 inhibited the migratory and invasive abilities of both $\mathrm{SiHa}$ and $\mathrm{C} 33 \mathrm{~A}$ cells.
Furthermore, the increased migratory and invasive abilities of SiHa and C33A cells induced by miR-96 were also abolished by CAV-1 overexpression, as indicated by the co-transfection of miR-96 and CAV-1 (Fig. 5C and D). The western blot analysis results suggested that the overexpression of $\mathrm{CAV}-1$ inhibited the activation of the Akt signaling pathway (Fig. 5E and F). 


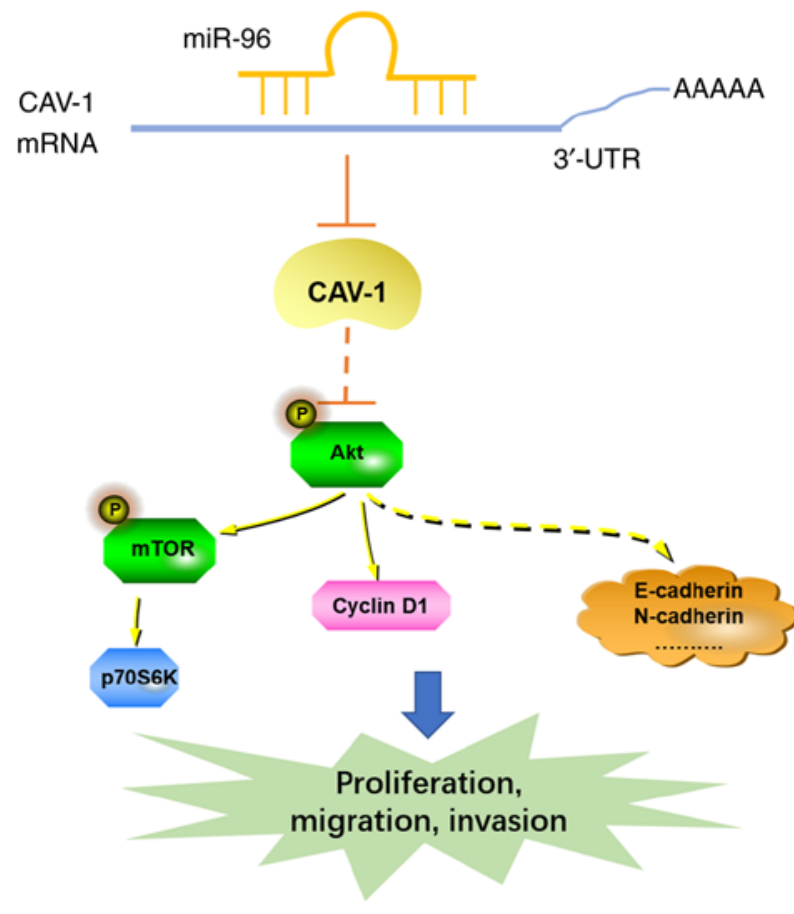

Figure 6. Schematic diagram of the miR-96/CAV-1/Akt/mTOR signaling pathway in cervical cancer cell proliferation, migration and invasion. miR-96, microRNA-96; CAV-1, caveolin 1; p-, phosphorylated; 3'UTR, 3'untranslated region; E-cadherin, epithelial cadherin; $\mathrm{N}$-cadherin, neural cadherin.

Further, the upregulation of p-Akt and p-mTOR induced by miR-96 was also demonstrated to be rescued by CAV-1 co-transfection in SiHa cells (Fig. 5E and F). Collectively, the present results suggested that miR-96 enhances cell proliferation, migration and invasion by targeting CAV-1, which modulates the Akt/mTOR signaling pathway in cervical cancer (Fig. 6).

\section{Discussion}

Previous studies have demonstrated that miR-96 is associated with tumorigenesis by acting as an oncogene or a tumor suppressor, depending on the tissue type $(18,21)$. The present results suggested that miR-96 was associated with the growth and metastatic potential of $\mathrm{HPV}^{+}$and $\mathrm{HPV}^{-}$cervical cancer cells. Overexpression of miR-96 was identified to facilitate the proliferative, migratory and invasive abilities of $\mathrm{SiHa}$ and C33A cells. Moreover, Ma et al (22) showed that miR-96 could promote the proliferation of Hela cell by silencing protein tyrosine phosphatase non-receptor type 9 , which is in concordance with the results of the present study. Therefore, miR-96 may serve an oncogenic role in the progression of cervical cancer, and it may be used as a potential target for cervical cancer therapy.

The role of CAV-1 in cancer biology is controversial, with some previous studies suggesting that CAV-1 plays a negative regulatory role in tumor metastasis $(24,25)$. Overexpression of CAV-1 inhibits the migration of HeLa cells via its caveolin scaffolding domain to regulate cell signaling (30). However, a recent study showed that silencing CAV-1 can inhibit the proliferation of lung adenocarcinoma $\mathrm{H} 522$ cells, but overexpression of CAV-1 is associated with a worse overall survival (25). Moreover, previous studies have suggested that the expression level of CAV-1 is tissue-type dependent, as CAV-1 is downregulated in ovarian cancer and colon cancer, but upregulated in bladder cancer and breast cancer $(25,31)$. Furthermore, the function and expression level of CAV-1 may be correlated with the grade and stage of cancer $(25,32,33)$. CAV-1 often acts as a tumor suppressor with decreased expression levels during the early stage of cancer progression, but enhances tumor aggressive and metastatic functions in the advanced stage of cancer $(25,32,33)$. Therefore, CAV-1 may serve a role as a tumor promotor and as a tumor suppressor in cancer progression. The present results suggested that CAV-1 acts as a tumor suppressor in cervical cancer, and that CAV-1 overexpression may inhibit cell proliferation, migration and invasion. Moreover, it was demonstrated that miR-96 can directly bind to CAV-1 mRNA and negatively regulate its expression in cervical cancer cells. Furthermore, overexpression of CAV-1 could reverse the increase in cell proliferation, migration and invasion induced by miR-96. Collectively, the present results indicated that miR-96 increased the growth and metastatic potential of cervical cancer by targeting CAV-1.

In the present study, it was identified that the overexpression of miR-96 upregulated the Akt/mTOR signaling pathway in cervical cancer cells. The Akt/mTOR signaling pathway serves essential roles in cancer cell activity, and is an important therapeutic target in cancer treatment (34). Yang et al (35) reported that miR-96 mimics activate the Akt/GSK-3 $\beta /$ $\beta$-catenin signaling pathway in hepatocellular carcinoma, which is involved in the carcinogenic effect of miR-96. Moreover, Song et al (36) showed that miR-96 may inhibit the expression of Forkhead Box O1 (FOXO1) via the upregulation the Akt/FOXO1/Bim signaling pathway in papillary thyroid carcinoma cells. Therefore, the present results indicated that the Akt/mTOR signaling pathway may be involved in the oncogenic role of miR-96 in cervical cancer. The effect of miR-96 on the transport of CAV-1 and the association between the miR-96/CAV-1/Akt signaling pathway should be further investigated in future studies.

In summary, the present results suggested that miR-96 exerted a carcinogenic effect by targeting CAV-1 in both $\mathrm{HPV}^{+}$and $\mathrm{HPV}^{-}$cervical cancer cells, and that the Akt/mTOR signaling pathway may be involved in this process. Therefore, miR-96 may function as a potential target in cervical cancer therapy. However, the present study only investigated the role of miR-96 in the biological function of $\mathrm{HPV}^{+}$and $\mathrm{HPV}^{-}$cervical cancer cells, thus further study is required to investigate the role of miR-96 in the progression of cervical cancer in vivo.

\section{Acknowledgements}

Not applicable.

\section{Funding}

No funding was received.

\section{Availability of data and materials}

The datasets used and/or analyzed during the present study are available from the corresponding author on reasonable request. 


\section{Authors' contributions}

YC, CL and BX conceived and designed the experiments. All authors performed the experiments. SC, YZ and SZ analyzed the data. YC wrote the manuscript. All authors read and approved the final manuscript.

\section{Ethics approval and consent to participate}

Not applicable.

\section{Patient consent for publication}

Not applicable.

\section{Competing interests}

The authors declare that they have no competing interests.

\section{References}

1. Small W Jr, Bacon MA, Bajaj A, Chuang LT, Fisher BJ, Harkenrider MM, Jhingran A, Kitchener HC, Mileshkin LR, Viswanathan AN and Gaffney DK: Cervical cancer: A global health crisis. Cancer 123: 2404-2412, 2017.

2. Xiao B, Xie Z, Guo L, Wu J and Zhang H: Stomatin-like protein 2 expression is associated with clinical survival in patients with cervical cancer. Int J Clin Exp Pathol 8: 1804-1809, 2015.

3. de Sanjose S, Quint WG, Alemany L, Geraets DT, Klaustermeier JE, Lloveras B, Tous S, Felix A, Bravo LE, Shin HR, et al: Human papillomavirus genotype attribution in invasive cervical cancer: A retrospective cross-sectional worldwide study. Lancet Oncol 11: 1048-1056, 2010.

4. Lecuru F, Bats A, Mathevet P, Querleu D, Leblanc E, Darai PM, Marret H, Collin C, Chatellier G and Gilaizeau F: Impact of sentinel lymph node biopsy on staging of early cervical cancer: Results of a prospective, multicenter study. J Clin Oncol 27: CRA5506, 2009.

5. Zhang Z, Wang J, Li J, Wang X and Song W: MicroRNA-150 promotes cell proliferation, migration, and invasion of cervical cancer through targeting PDCD4. Biomed Pharmacother 97: 511-517, 2017.

6. Gregory RI and Shiekhattar R: MicroRNA biogenesis and cancer. Cancer Res 65: 3509-3512, 2011.

7. Esquela-Kerscher A and Slack F: Oncomirs-microRNAs with a role in cancer. Nat Rev Cancer 6: 259-269, 2006.

8. Li GC, Cao XY, Li YN, Qiu YY, Li YN, Liu XJ and Sun XX: MicroRNA-374b inhibits cervical cancer cell proliferation and induces apoptosis through the p38/ERK signaling pathway by binding to JAM-2. J Cell Physiol 233: 7379-7390, 2018

9. Wang F, Shan S, Huo Y, Xie Z, Fang Y, Qi Z, Chen F, Li Y and Sun B: MiR-155-5p inhibits PDK1 and promotes autophagy via the mTOR pathway in cervical cancer. Int J Biochem Cell Biol 99: 91-99, 2018

10. Kouji B, Iida M, Yanokura M, Kisu I, Iwata T, Tominaga E, Tanaka K and Aoki D: MicroRNA in cervical cancer: OncomiRs and tumor suppressor miRs in diagnosis and treatment. ScientificWorldJournal 2014: 178075, 2014.

11. Garzon R, Marcucci G and Croce CM: Targeting microRNAs in cancer: Rationale, strategies and challenges. Nat Rev Drug Discov 9: 775-789. 2010.

12. Rasras S, Zibara K, Vosughi T and Zayeri Z: Genetics and epigenetics of glioblastoma: Therapeutic challenges. Clin Cancer Invest J 7: 43-49, 2018.

13. Takahashi RU, Prieto Vila M, Kohama I and Ochiya T: Development of microRNA-based therapeutic approaches for cancer patients. Cancer Sci 110: 1140-1147, 2019.

14. Chakraborty C, Sharma AR, Sharma G, Sarkar BK and Lee SS The novel strategies for next-generation cancer treatment: miRNA combined with chemotherapeutic agents for the treatment of cancer. Oncotarget 9: 10164-10174, 2018.
15. Mittal A, Chitkara D, Behrman SW and Mahato RI: Efficacy of gemcitabine conjugated and miRNA-205 complexed micelles for treatment of advanced pancreatic cancer. Biomaterials 35: 7077-7087, 2014

16. Shi S, Han L, Deng L, Zhang Y, Shen H, Gong T, Zhang Z and Sun X: Dual drugs (microRNA-34a and paclitaxel)-loaded functional solid lipid nanoparticles for synergistic cancer cell suppression. J Control Release 194: 228-237, 2014.

17. Testoni D, Hayashi M, Cohen-Wolkowiez M, Benjamin DK Jr, Lopes RD, Clark RH, Benjamin DK and Smith P: Late-onset bloodstream infections in hospitalized term infants. Pediatr Infect Dis J 33: 920-923, 2014.

18. Xie W, Sun F, Chen L and Cao X: MiR-96 promotes breast cancer metastasis by suppressing MTSS1. Oncol Lett 15:3464-3471, 2018.

19. Yang S, Chen Z, Fan D, Zhang R, Zhang Y and Wu S: MiR-182-5p and miR-96-5p increased hepatocellular carcinoma cell mobility, proliferation and cisplatin resistance partially by targeting RND3. RSC Advances 8: 34973-34983, 2018.

20. Fendler A, Jung M, Stephan C, Erbersdobler A, Jung K and Yousef GM: The antiapoptotic function of miR-96 in prostate cancer by inhibition of FOXO1. PLoS One 8: e80807, 2013.

21. He C, Zhang Q, Gu R, Lou Y and Liu W: miR-96 regulates migration and invasion of bladder cancer through epithelial-mesenchymal transition in response to transforming growth factor- $\beta 1$. J Cell Biochem 119: 7807-7817, 2018.

22. Ma X, Shi W, Peng L, Qin X and Hui Y: MiR-96 enhances cellular proliferation and tumorigenicity of human cervical carcinoma cells through PTPN9. Saudi J Biol Sci 25: 863-867, 2018.

23. Sternberg PW and Schmid SL: Caveolin, cholesterol and Ras signalling. Nat Cell Biol 1: E35-E37, 1999.

24. Yeong J, Thike AA, Ikeda M, Lim JCT, Lee B, Nakamura S, Iqbal $J$ and Tan PH: Caveolin-1 expression as a prognostic marker in triple negative breast cancers of Asian women. J Clin Pathol 71: 161-167, 2017.

25. Duregon E, Senetta R, Bertero L, Bussolati B, Annaratone L, Pittaro A, Papotti M, Marchiò C and Cassoni P: Caveolin 1 expression favors tumor growth and is associated with poor survival in primary lung adenocarcinomas. Tumor Biol 39: $101042831769431,2017$.

26. Zhou W,He L, Dai Y,Zhang Y, Wang J and Liu B: MicroRNA-124 inhibits cell proliferation, invasion and migration by targeting CAV1 in bladder cancer. Exp Ther Med 16: 2811-2820, 2018.

27. Butz H, Szabó PM, Khella HW, Nofech-Mozes R, Patocs A and Yousef GM: miRNA-target network reveals miR-124 as a key miRNA contributing to clear cell renal cell carcinoma aggressive behaviour by targeting CAV1 and FLOT1. Oncotarget 6: 12543-12557, 2015.

28. Livak KJ and Schmittgen TD: Analysis of relative gene expression data using real-time quantitative PCR and the 2(-Delta Delta C(T)) method. Methods 25: 402-408, 2001.

29. Li JH, Liu S, Zhou H, Qu LH and Yang JH: Starbase v2.0: Decoding miRNA-ceRNA, miRNA-ncRNA and protein-RNA interaction networks from large-scale clip-seq data. Nucleic Acids Res 42 (Database issue): D92-D97, 2014.

30. Okada S, Raja SA, Okerblom J, Boddu A, Horikawa Y, Ray S, Okada H, Kawamura I, Murofushi Y, Murray F and Patel HH: Deletion of caveolin scaffolding domain alters cancer cell migration. Cell Cycle 18: 1268-1280, 2019.

31. Senetta R, Trevisan E, Rudà R, Maldi E, Molinaro L, Lefranc F, Chiusa L, Lanotte M, Soffietti R and Cassoni P: Caveolin 1 expression independently predicts shorter survival in oligodendrogliomas. J Neuropathol Exp Neurol 68: 425-431, 2009.

32. Shatz $\mathrm{M}$ and Liscovitch M: Caveolin-1: A tumor-promoting role in human cancer. Int J Radiat Biol 84: 177-189, 2008.

33. Goetz J, Lajoie P, M Wiseman S and Nabi I: Caveolin-1 in tumor progression: The good, the bad and the ugly. Cancer Metastasis Rev 27: 715-735, 2008.

34. Martini M, De Santis M, Braccini L, Gulluni F and Hirsch E: PI3K/AKT signaling pathway and cancer: An updated review. Ann Med 46: 372-383, 2014.

35. Yang N, Zhou J, Li Q, Han F and Yu Z: miR-96 exerts carcinogenic effect by activating AKT/GSK-3 $\beta / \beta$-catenin signaling pathway through targeting inhibition of FOXO1 in hepatocellular carcinoma. Cancer Cell Int 19: 38, 2019.

36. Song HM,Luo Y,Li DF, Wei CK, Hua KY, Song JL, Xu H, Maskey N and Fang L: MicroRNA-96 plays an oncogenic role by targeting FOXO1 and regulating AKT/FOXO1/Bim pathway in papillary thyroid carcinoma cells. Int J Clin Exp Pathol 8: 9889-9900, 2015. 